\title{
Analytic Combinatorics of the Mabinogion Urn
}

\author{
Philippe Flajolet ${ }^{1}$ and Thierry Huillet ${ }^{2}$ \\ ${ }^{1}$ Algorithms Project, INRIA Rocquencourt, Domaine de Voluceau, F-78153, Le Chesnay, France Phi l ippe. Fla jolet@inria. fr. \\ ${ }^{2}$ Laboratoire de Physique Théorique et Modélisation, CNRS-UMR 8089 and Université de Cergy-Pontoise, 2 Avenue \\ Adolphe Chauvin, F-95302, Cergy-Pontoise, France Thierry.Huilletau-cergy. fr.
}

\begin{abstract}
The Mabinogion urn is a simple model of the spread of influences amongst versatile populations. It corresponds to a non-standard urn with balls of two colours: each time a ball is drawn, it causes a ball of the other kind to switch its colour. The process stops once unanimity has been reached. This note provides analytic expressions describing the evolution of the Mabinogion urn, based on a time-reversal transformation applied to the classical Ehrenfest urn. Consequences include a precise asymptotic analysis of the stopping-time distribution-it is asymptotically normal in the "unfair" case and akin to an extreme-value (double exponential) distribution in the "fair" case-as well as a characterization of the exponentially small probability of reversing a majority.
\end{abstract}

Keywords: Analytic combinatorics, asymptotic analysis, urn models.

\section{Introduction}

The purpose of this paper is to introduce and study a simple model of the stochastic spread of influence in populations. Say a country consists of a population of $N$ sheep, each of which can, at any given time, be in either one of two states of mind, denoted by $A$ and $B$. At discrete instants $1,2,3, \ldots$, a randomly chosen sheep in the population bleats in accordance with its current state of mind: if this sheep bleats $A$ [aah], then one of the $B$-sheep changes to state $A$; if this sheep bleats $B$ [eeh], then one of the $A$-sheep changes to state $B$. The process stops when unanimity has been reached; that is, all sheep are in one and the same state. We call this model the Mabinogion urn process, following Williams [38, §15.3], who analysed a rather specific "controlled" version. The name itself comes from a Welsh medieval tale, The Mabinogion, where one excerpts the following piece:

"And he came towards a valley, through which ran a river; and the borders of the valley were wooded, and on each side of the river were level meadows. And on one side of the river he saw a flock of white sheep, and on the other a flock of black sheep. And whenever one of the white sheep bleated, one of the black sheep would cross over and become white; and when one of the black sheep bleated, one of the white sheep would cross over and become black."

(See http://www.sacred-texts.com/neu/celt/mab/mab10.htm.) The problem is to determine the stopping time of the process as well as the probability that unanimity of either type is eventually reached, when given a certain initial condition of the population.

1365-8050 @ 2008 Discrete Mathematics and Theoretical Computer Science (DMTCS), Nancy, France 
The Mabinogion urn was studied by us during the 2007 presidential election campaign in France, where two candidates were running for president. To model this situation, one can take $N=6 \cdot 10^{7}$. Say one sheep bleats every second. If initially, the population is equally divided between the two candidates, what is the expected time needed to reach eventual unanimity? We shall determine the answer to be about 8 years. If the initial proportions of $A$-sheep correspond to a minority of $47 \%, 49 \%$, and $49.9 \%$, what is the probability of an eventual unanimity of type A? Answer: about $10^{-46,000}, 10^{-5,000}$, and $10^{-50}$, respectively. These are typical questions addressed in this paper.

The Mabinogion model described above fits into the category of (generalized) Pólya urn models, for which Johnson and Kotz [21] provide an elementary introduction. An urn may contain balls of two colours, say $A$ and $B$, and a $2 \times 2$ matrix

$$
\left(\begin{array}{ll}
\alpha & \beta \\
\gamma & \delta
\end{array}\right), \quad \alpha, \beta, \gamma, \delta \in \mathbb{Z},
$$

is given. At discrete instants, $1,2, \ldots, n$, a random ball in the urn is chosen and its colour is inspected: if that colour is $A$, then $\alpha$ balls of type $A$ and $\beta$ balls of type $B$ are inserted; if the colour is $B$, then $\gamma$ balls of type $A$ and $\delta$ balls of type $B$ are inserted. Negative entries in the matrix are interpreted as taking out balls of the corresponding colour. The initial composition $\left(a_{0}, b_{0}\right)$ of the urn at time 0 being fixed, the goal is to characterize its (random) composition $\left(A_{n}, B_{n}\right)$ at time $n$.

The Mabinogion model is then seen to be a Pólya urn corresponding to the matrix

$$
\mathcal{M}=\left(\begin{array}{cc}
1 & -1 \\
-1 & 1
\end{array}\right)
$$

The presence of off-diagonal entries that are negative precludes an appeal to the standard theory of urn processes, which is well-exemplified by Janson's recent studies [19,20]. Our approach consists in relating the evolution of the Mabinogion urn to that of the classical Ehrenfest ury ${ }^{(\mathrm{i})}$, which is described by the matrix

$$
\mathcal{E}=\left(\begin{array}{cc}
-1 & 1 \\
1 & -1
\end{array}\right)
$$

and we do so by means of a time-reversal transformation (note that $\mathcal{M}=-\mathcal{E}$ ). Although the stochastic evolutions of both urns are radically different, the analytic formulae available for the Ehrenfest urn can be recycled: our treatment starts precisely from this observation. The methods we develop are entirely analytic, being based on explicit integral representations of the quantities of interest and classical asymptotic methods like the Laplace and saddle-point methods-our treatment follows the general principles of analytic combinatorics, as presented in the forthcoming book [13]. Our results then supplement the recent study by Flajolet, Dumas, and Puyhaubert [12], which is dedicated to exactly solvable models of urn process theory, by providing a new urn that admits of explicit formulae.

The Mabinogion urn can also, broadly speaking, be viewed as a special process with reinforcement in the sense of Pemantle's survey [30]. In this context, it appears as a peaceful alternative to the OK Corral urn defined by the matrix,

$$
\mathcal{O}=\left(\begin{array}{cc}
0 & -1 \\
-1 & 0
\end{array}\right)
$$

(i) The Ehrenfest urn [7] serves to model particle (or heat) transfer between two chambers. As it is well known, it has played an important historical rôle in resolving the apparent contradiction between recurrence in Markov chains and irreversibility in statistical thermodynamics [5] 22]. 

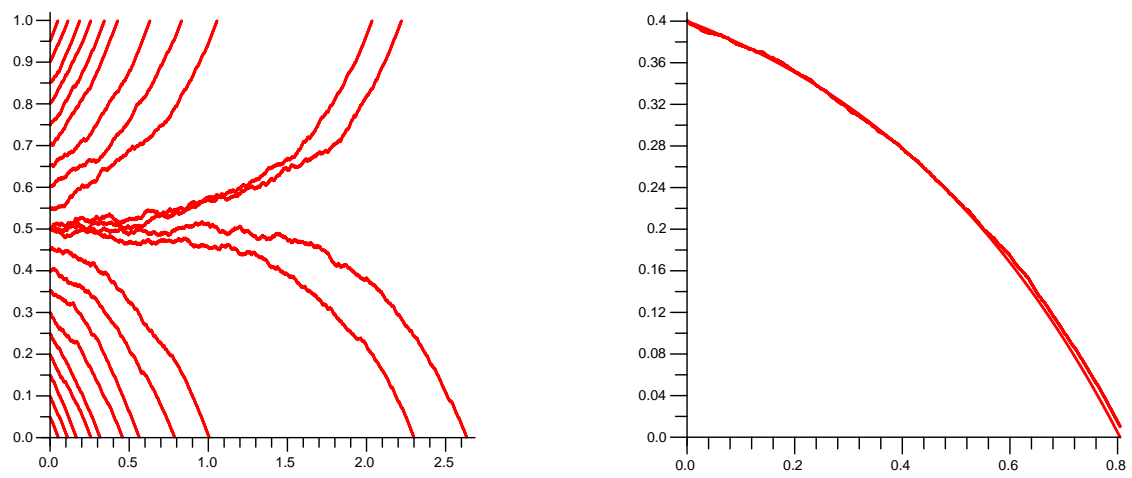

Fig. 1: Left: Trajectories of the Mabinogion urn for $N=1000$ and sizes of the initial $A$-population ranging from 50 to 950 , with four simulations for the fair case $k=N / 2=500$. Right: A sample path of the urn for $N=10^{4}$, initialized with 4000 elements of type $A$, compared to the deterministic approximation 2). (Horizontal axis: time $n$ in units of $N$; vertical axis: size $A_{n}$ of the $A$-population in proportion to $N$.)

under which a $B$-sheep disappears (is killed!) whenever an $A$-sheep bleats, and vice-versa. That urn was introduced by Williams and McIlroy in [39] and further studied by Kingman in [25], who observed that it constitutes one of the simplest cases of Lanchester's theory of conflicts [26]. Kingman and Volkov [27] (see also [12, 18, 31]) then showed that the OK Corral urn can be described as a time-reversed version of the classical Friedman urn, determined by the matrix

$$
\mathcal{F}=\left(\begin{array}{ll}
0 & 1 \\
1 & 0
\end{array}\right),
$$

which can itself be viewed as modelling "adverse-campaign effects" [14]. Our reduction of the Mabinogion urn to the Ehrenfest urn parallels Kingman and Volkov's reduction (note that $\mathcal{O}=-\mathcal{F}$ ) and was clearly inspired by it.

\section{The physics of the Mabinogion urn}

To get a feel of what goes on, we started by simulating the Mabinogion urn for various values of the initial size of the $A$-population (at time 0 ) and for some fixed total population $N$; see Figure 1 . Several properties of the model are already apparent there.

Consider first, for large $N$, the evolution of the urn initialized with $k=\lfloor a N\rfloor$ balls of type $A$, when $a<\frac{1}{2}$. The $A$-group is at a disadvantage initially, and each transition of the urn reinforces the chances of an $A$-element to become a $B$. The $A$-population must be, with high probability, doomed to extinction, with the time for this to happen being $O(N)$ in probability. Indeed, with $A_{n}$ the size of the $A$-population at time $n$, consider the renormalized quantity

$$
X(t):=\frac{1}{N} \mathbb{E}\left[A_{\lfloor N t\rfloor}\right] .
$$


Heuristically, for large values of $N$, the evolution of the process should be well approximated by the differential equation

$$
\frac{d}{d t} X(t)=2 X(t)-1, \quad X(0)=a,
$$

whose solution is

$$
X(t)=\frac{1}{2}-e^{2 t}\left(a-\frac{1}{2}\right)
$$

In particular, this curve hits the horizontal line at abscissa

$$
\tau(a)=\frac{1}{2} \log \frac{1}{1-2 a},
$$

leading us to infer for the mean value of the time $T$ to reach absorption:

$$
\mathbb{E}\left[T_{\lfloor a N\rfloor}\right] \sim \tau(a) N .
$$

For instance, for $a=0.25$ and $a=0.4$, we expect the $A$-extinction time to be close to $0.35 \mathrm{~N}$ and $0.8 \mathrm{~N}$, respectively. Figure 1 [right] shows the approximation to be stunningly close to reality, in the case $a=0.4$.

For an initial size $a>\frac{1}{2}$, it is symmetrically the $B$ s that are doomed to disappear after $O(N)$ steps. That the $A$ s could still win can only occur as a long sequence of abnormally favorable choices, corresponding in essence to a large deviation situation for a sequence of Bernoulli trials. We may thus expect the probability of reversing the opinion of a majority (which initially dominates by a fixed percentage) to be exponentially small.

For the fair Mabinogion urn where $a=\frac{1}{2}$, there are obviously equal chances for the $A$ or $B$ to win. However, as is apparent from Figure 1 [left], where four such trajectories are plotted, the time till absorption becomes appreciably longer. There is a prolonged period of exchanges of influences, till eventually irregularities in the distribution of a (nearly) fair coin give a definite advantage to one of the two groups, which will from then on follow a deterministic trajectory. Indeed, we shall quantify the absorption time in that case to be $O(N \log N)$ in probability.

In the sequel, we precisely justify these empirical observations and provide explicit asymptotic forms for the various quantities involved. Everything is based on a combinatorial duality between the Mabinogion urn and the Ehrenfest urn, which is detailed in Section 3 , resulting in Theorem 1 . We then analyse the "unfair" Mabinogion urn in Section 4, which corresponds to a fixed imbalance between the initial sizes of the two populations. We prove in this case that the probability of reversing the majority is exponentially small, with an explicit rate provided by Theorem 2 Also, the time till absorption is on average $O(N)$, with a standard deviation of $O(\sqrt{N})$ and a limit law of the Gaussian type; see Theorem 3 For the "fair" Mabinogion urn, examined in Section 5, the scaling is different: the time till absorption turns out to be $O(N \log N)$ on average, with a limit distribution akin to an extreme-value statistics; see Proposition 2 and Theorem 4

\section{Analytic solution of the Mabinogion urn}

The main purpose of this section is to provide explicit formulae for the probability distribution of the stopping time of the Mabinogion urn process (Theorem 1 below). These arise, via a duality principle 
presented in Subsection 3.1, from generating function solutions associated to the Ehrenfest urn, which are derived in Subsection 3.2 Such a generating function approach to the Ehrenfest urn departs from the standard linear algebra methods introduced by Kac [22] and followed by most subsequent authors.

First a few notations. Under the Mabinogion model $\mathcal{M}$, the size of the population (of sheep, balls) remains a constant, which we consistently denote by $N$. At time $n$, i.e., after $n$ transitions of the Markov chain, the urn contains $A_{n}$ (respectively $B_{n}$ ) elements of type $A$ (respectively, of type $B$ ) and the (random) vector $\left(A_{n}, B_{n}\right)$ is called the urn composition at time $n$. The initial conditions are written as

$$
A_{0}=k, \quad B_{0}=N-k
$$

The main quantity of interest is the random variable $T \equiv T_{k}$ representing the time needed to reach absorption with all elements being of type $B$, and we agree that $T=+\infty$ in case the system stabilizes with all elements in state $A$. This quantity will be called the $A$-extinction time. It depends on $N$ as well as $k$, the event $(T<+\infty)$ corresponding to elements of type $B$ winning the game under the election metaphor. We state:

Theorem 1 (Extinction time distribution for $\mathcal{M}$ ) The probability distribution of the A-extinction time for the Mabinogion urn initialized with $\left(A_{0}, B_{0}\right)=(k, N-k)$ satisfies for all $n \in \mathbb{Z}_{\geq 0}$

$$
\mathbb{P}(T=n+1)=\frac{N-1}{N^{n+1}}\left(\begin{array}{c}
N-2 \\
k-1
\end{array}\right) n !\left[z^{n}\right](\sinh z)^{k-1}(\cosh z)^{N-k-1} .
$$

An equivalent formulation is

$$
\mathbb{P}(T=n+1)=\left(1-\frac{1}{N}\right) \frac{n !}{2^{N-2}} \sum_{\ell=1}^{N-1} K_{k-1, \ell-1}^{(N-1)}\left(1-\frac{2 \ell}{N}\right)^{n}
$$

where the "Kac coefficients" are given by

$$
K_{k, \ell}^{(N+1)}=\left[y^{\ell}\right](1+y)^{N-k}(1-y)^{k}=\sum_{j}(-1)^{j}\left(\begin{array}{c}
N-k \\
\ell-j
\end{array}\right)\left(\begin{array}{c}
k \\
j
\end{array}\right) .
$$

There the notation $\left[z^{n}\right] f(z)$, which is common in combinatorial mathematics [17], represents the coefficient of $z^{n}$ in the expansion of $f$ at 0 ; in other words:

$$
f_{n} \equiv\left[z^{n}\right] \sum_{m} f_{m} z^{m}
$$

In fact, our subsequent developments rely on the generating function expression (3). (The equivalent form (4), which is a double alternating sum, is given for completeness. It plainly results from the identity

$$
(\sinh z)^{k}(\cosh z)^{N-k}=2^{N} e^{N z}\left(1-e^{-2 z}\right)^{k}\left(1+e^{-2 z}\right)^{N-k},
$$

upon expanding in powers of $e^{z}$, then in powers of $z$.) Theorem 1 results directly from Lemmas 1 and 2 below: see the discussion surrounding Equation 13 at the end of this section. 


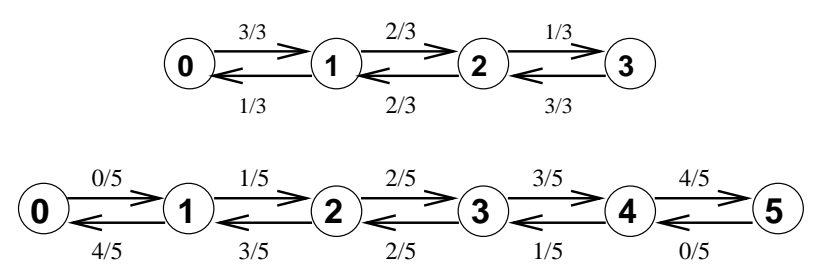

Fig. 2: The transition diagrams of the Ehrenfest urn with $N=3$ particles (top) and of the Mabinogion urn with $N+2=5$ sheep (bottom) illustrate the duality between the two models under time reversal.

\subsection{The Ehrenfest-Mabinogion duality}

The Mabinogion urn with $N$ balls, $\mathcal{M}[N]$, is a Markov chain with transition probabilities

$$
\mathbb{P}\left(A_{n+1}=\ell+1 \mid A_{n}=\ell\right)=\frac{\ell}{N}, \quad \mathbb{P}\left(A_{n+1}=\ell-1 \mid A_{n}=\ell\right)=1-\frac{\ell}{N} \quad(0<\ell<N) .
$$

For the Ehrenfest urn with $N$ balls, $\mathcal{E}[N]$, the transition probabilities are

$$
\mathbb{P}\left(\widetilde{A}_{n+1}=\ell+1 \mid \widetilde{A}_{n}=\ell\right)=1-\frac{\ell}{N}, \quad \mathbb{P}\left(\widetilde{A}_{n+1}=\ell-1 \mid \widetilde{A}_{n}=\ell\right)=\frac{\ell}{N} \quad(0 \leq \ell \leq N),
$$

where $\widetilde{A}_{n}$ represents the number of balls of the first type at time $n$. There is an obvious symmetry in these formula, which is evident in the transition diagrams of Figure 2, adapted from [12, §6]. There is also a difference due to boundary conditions (ii) in (6) and (7), which explains the fact that the correspondence links $\mathcal{E}[N]$ to $\mathcal{M}[N+2]$. Precisely, we have, regarding transitions between states, i.e., sizes of the $A-$ population:

Lemma 1 The Mabinogion and Ehrenfest urns are related through:

$$
\mathbb{P}_{\mathcal{M}[N+2]}(\sigma+1 \mapsto 0 \text { in } n+1 \text { steps })=\frac{N^{n}(N+1)}{(N+2)^{n+1}} \mathbb{P}_{\mathcal{E}[N]}(0 \mapsto \sigma \text { in } n \text { steps }) \text {. }
$$

Proof: Consider the directed multigraph $\Gamma[N]$ whose vertices are the set $\{0,1, \ldots, N\}$ and whose edges are all the possible pairs of the form $(j, j+1)$ and $(j, j-1)$. Multiplicities are attached to edges, the multiplicity of an edge $(j, j+1)$ being $N-j$, that of an edge $(j, j-1)$ being $j$. Diagrammatically, for $N=3$ :

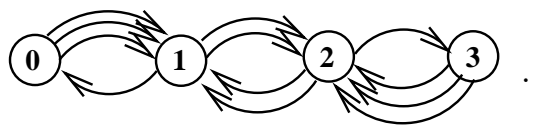

Let $W_{\sigma, n}^{(N)}$ be the number of paths in $\Gamma[N]$ comprised of $n$ steps, starting from vertex 0 , and ending in vertex $\sigma$. From the definition of the Markov chain $\mathcal{E}[N]$ that underlies the Ehrenfest urn, we have

$$
\mathbb{P}_{\mathcal{E}[N]}(0 \mapsto \sigma \text { in } n \text { steps })=\frac{1}{N^{n}} \cdot W_{\sigma, n}^{(N)},
$$

(ii) Extreme states are reflecting for the $\mathcal{E}$-urn, absorbing for the $\mathcal{M}$-urn. 
since the probability of any particular path is $1 / N^{n}$. Similarly, consider a sample path of length $n+1$ in the Markov chain $\mathcal{M}[N+2]$, starting from state $\sigma+1$ and ending in state 0 . Its last step has probability $(N+1) /(N+2)$. The path stripped of its last step is then such that its mirror image (this corresponds to a time reversal transformation) is a path in $\Gamma[N]$ from 0 to $\sigma$, each such stripped path having weight $1 /(N+2)^{n}$. Thus, we have

$$
\mathbb{P}_{\mathcal{M}[N+2]}(\sigma+1 \mapsto 0 \text { in } n+1 \text { steps })=\frac{N+1}{N+2} \cdot \frac{1}{(N+2)^{n}} \cdot W_{\sigma, n}^{(N)} .
$$

The comparison of the last two displayed equations proves the statement.

\subsection{Analysis of the Ehrenfest urn}

Following Kac, the analysis of the Ehrenfest urn is traditionally developed from an explicit diagonalization of the $(N+1) \times(N+1)$ "Kac matrix",

$$
\mathrm{Kac}^{[N+1]}=\left(\begin{array}{cccccc}
0 & N & & & & \\
1 & 0 & N-1 & & & \\
& 2 & 0 & N-2 & & \\
& & & \ddots & \ddots & \ddots \\
& & & & N & 0
\end{array}\right)
$$

which, up to normalization, is the matrix of the Markov chain $\mathcal{E}[N]$. Our approach relies instead on properties exponential generating functions (EGFs). We believe that it provides the simplest possible solution (via just two equations, 11 and $(12$ below) to the Ehrenfest urn, one on which our subsequent developments are entirely based.

Lemma 2 The Ehrenfest urn with $N$ balls satisfies

$$
\mathbb{P}_{\mathcal{E}[\mathcal{N}]}(0 \mapsto \ell \text { in n steps })=\frac{n !}{N^{n}}\left(\begin{array}{c}
N \\
\ell
\end{array}\right)\left[z^{n}\right](\sinh z)^{\ell}(\cosh z)^{N-\ell}
$$

Proof: For the urn $\mathcal{E}[N]$, consider the $N$ (distinguishable) balls to be numbered by integers of $\{1, \ldots, N\}$. Now let $\mathrm{e}_{n}$ be 1 if $n$ is even and 0 otherwise, and set $\mathrm{o}_{n}=1-\mathrm{e}_{n}$. Under the initial condition $\left(\widetilde{A}_{0}, \widetilde{B}_{0}\right)=$ $(0, N)$, the probability of reaching at time $n$ the state where balls numbered 1 to $\ell$ are in state $A$, the other ones being in state $B$, is

$$
\pi_{n}=\frac{1}{N^{n}} \sum_{n_{1}+\cdots+n_{N}=n}\left(\begin{array}{c}
n \\
n_{1}, \ldots, n_{N}
\end{array}\right)\left(\mathrm{o}_{n_{1}} \cdots \mathrm{o}_{n_{\ell}}\right) \cdot\left(\mathrm{e}_{n_{\ell+1}} \cdots \mathrm{e}_{n_{N}}\right) .
$$

Reason: the multinomial counts the partitions of $\{1, \ldots, n\}$ into $N$ possibly empty subsets, where the $j$ th subset corresponds to the instants at which ball $j$ changes states; the product $\left(\mathrm{o}_{n_{1}} \cdots \mathrm{o}_{n_{\ell}}\right) \cdot\left(\mathrm{e}_{n_{\ell+1}} \cdots \mathrm{e}_{n_{N}}\right)$ restricts the summation to those cases where balls 1 to $\ell$ change an odd number of times, the other balls changing an even number of times. Now the key device is to appeal to exponential generating functions,

$$
\pi(z):=\sum_{n \geq 0} \pi_{n} \frac{z^{n}}{n !}, \quad \cosh (z)=\sum_{n \geq 0} \mathrm{e}_{n} \frac{z^{n}}{n !}, \quad \sinh (z)=\sum_{n \geq 0} \mathrm{o}_{n} \frac{z^{n}}{n !} .
$$


The convolution equation (11) then translates into the EGF relation

$$
\pi(z)=\left[(\sinh z)^{\ell}(\cosh z)^{N-\ell}\right]_{z \rightarrow z / N} .
$$

The statement finally follows upon introducing the multiplier $\left(\begin{array}{c}N \\ \ell\end{array}\right)$, which counts the possible choices for the identities of those $\ell$ balls that remain in state $A$.

We can now complete the proof of Theorem 1 as follows:

$$
\left\{\begin{aligned}
\mathbb{P}\left(T_{k}=n+1\right) & \equiv \mathbb{P}_{\mathcal{M}[N]}(k \mapsto 0 \text { in } n+1 \text { steps }) \\
& =\frac{(N-2)^{n}(N-1)}{N^{n+1}} \mathbb{P}_{\mathcal{E}[N-2]}(0 \mapsto k-1 \text { in } n \text { steps }) \\
& =\frac{N-1}{N^{n+1}}\left(\begin{array}{c}
N-2 \\
k-1
\end{array}\right) \cdot n !\left[z^{n}\right](\sinh z)^{k-1}(\cosh z)^{N-k-1} .
\end{aligned}\right.
$$

The first line expresses the definition of $T_{k}$. The second line results from the duality of Lemma 1 . The last line is a consequence of the analytic solution of the Ehrenfest urn in Lemma2.

Note 1. (i) Modern theories of combinatorial analysis [13, 15, 34], especially the "labelled product" construction, make it possible to write directly (12) as the EGF of ordered partitions having their first $k$ blocks even and the other blocks odd, this without ever a need to develop explicitly the convolution relations 11 . In this way other parameters of the Ehrenfest urn can be swiftly analysed [16].

(ii) The general analytic approach to balanced urn models developed in [12] is applicable. In that case the analysis of the Ehrenfest urn is reduced to properties of the linear partial-differential operator

$$
\mathfrak{D}=y \partial_{x}+x \partial_{y}
$$

and its associated non-linear ordinary differential system

$$
\{\dot{X}=Y, \quad \dot{Y}=X\}
$$

leading directly to hyperbolic functions.

(iii) The bridge to solutions expressed in terms of ordinary, rather than exponential, generating functions is provided by the Laplace transform. The former are amenable to Flajolet's theory of continued fractions [11]. One obtains in this way a combinatorial interpretation of the identity,

$$
\int_{0}^{\infty} e^{-t}(\cosh z t)^{-N} d t=\frac{1}{1-\frac{1 \cdot N z^{2}}{1-\frac{2 \cdot(N-1) z^{2}}{\ddots}}},
$$

first discovered by Stieltjes and Rogers [3, 16, 32, 36], themselves, to some extent, anticipated by Euler [8]. From there, or from the expanded forms (4), it is apparent that the spectrum of the matrix $\operatorname{Kac}^{[N+1]}$ is $\{-N,-N+$ $2, \ldots, N-2, N\}$. (Further interesting matrix connections are presented in 6, 9 .) 


\section{The unfair Mabinogion urn}

In accordance with previous notations, we consider the time $T$ till $A$-extinction of the Mabinogion urn $\mathcal{M}[N]$ (all the elements have become of $B$-type), given that the initial composition of the urn is $A_{0}=k$, $B_{0}=N-k$. We take $T=+\infty$ to mean that no $A$-extinction takes place: the $A \mathrm{~s}$ win! We adopt $k=\lfloor x N\rfloor$. In this section, the quantity $x$ will invariably assume a fixed value different from $1 / 2$ : this is the unfair case. Proceeding according to increasing calculational complexity, we first show that the probability of reversing a majority (i.e., reaching $A$-extinction, when $\frac{1}{2}<x<1$ ) is quantifiable and exponentially small (Theorem 2); then that, for $x<1 / 2$, the time till $A$-extinction is asymptotically normal (Theorem 3 ).

\subsection{Reversing a majority}

If $f(z)=\sum f_{n} z^{n} / n$ ! is an exponential generating function, the Laplace transform integral provides a representation of the corresponding ordinary generating function:

$$
\sum f_{n} u^{n}=\int_{0}^{\infty} e^{-z} f(z u) d z
$$

On the other hand, the probabilities of the extinction time are described by Equation (3) of Theorem 1 as coefficients of an exponential generating function. The probability generating function (PGF) of the finite part of $T$, which is

$$
\phi_{N, k}(u):=\mathbb{E}\left(u^{T}\right) \equiv \sum_{n \geq 0} \mathbb{P}(T=n+1) u^{n+1},
$$

equals then, by (15):

$$
\phi_{N, k}(u)=u \frac{N-1}{N}\left(\begin{array}{c}
N-2 \\
k-1
\end{array}\right) \int_{0}^{\infty} e^{-z} \sinh \left(\frac{u z}{N}\right)^{k-1} \cosh \left(\frac{u z}{N}\right)^{N-k-1} d z .
$$

This representation is convergent when $|\Re(u)|<N /(N-2)$, for any $k \in 1, \ldots, N-1$. It can in particular be used to analyse the probability $\omega_{N, k}$ of $A$-extinction. This probability, obtained by setting $u=1$ in (17), is:

$$
\omega_{N, k} \equiv \phi_{N, k}(1)=\frac{N-1}{N}\left(\begin{array}{c}
N-2 \\
k-1
\end{array}\right) \int_{0}^{\infty} e^{-z} \sinh \left(\frac{z}{N}\right)^{k-1} \cosh \left(\frac{z}{N}\right)^{N-k-1} d z .
$$

We have, when $x=k / N$ satisfies $x>1 / 2$ :

Theorem 2 (Unfair urn: majority reversal) Consider the Mabinogion urn $\mathcal{M}[N]$ with initial composition $\left(A_{0}, B_{0}\right)=(k, N-k)$ and $k=\lfloor x N\rfloor$, where $\frac{1}{2}<x<1$. The probability $\omega_{N, k}$ of eventual $A$-extinction is exponentially small:

$$
-\lim _{N \rightarrow \infty} \frac{1}{N} \log \omega_{N,\lfloor x N\rfloor}=\log 2+x \log x+(1-x) \log (1-x) .
$$

Proof: With the the change of variables $y=e^{-2 z / N}$, the representation 18 is first expressed as

$$
\omega_{N, k}=2^{-N+1}(N-1)\left(\begin{array}{c}
N-2 \\
k-1
\end{array}\right) \int_{0}^{1}(1-y)^{k-1}(1+y)^{N-k-1} d y
$$


Set $\xi=k / N$, so that, for large $N$, the quantity $\xi$ is very nearly equal to $x$, and define

$$
J_{N}(\xi):=\int_{0}^{1}(1-y)^{\xi N}(1+y)^{(1-\xi) N} \frac{d y}{1-y^{2}} .
$$

The function $(1-y)^{\xi}(1+y)^{1-\xi}$ is decreasing over $(0,1)$, since $\xi>1 / 2$.

The estimation of $J_{N}$ is a textbook application of the Laplace method for integrals involving large powers, in the situation where the maximum of the integrand is located at one of the end-points-then only a small neighbourhood of that end-point is expected to contribute significantly. Indeed, let $\mathcal{E} \mathcal{S}(N)$ denote any function that is exponentially small in $N$ in the sense that

$$
h(N)=\mathcal{E} \mathcal{S}(N) \quad \text { iff } \quad h(N)=O\left(\exp \left(-C N^{D}\right)\right),
$$

for some $C, D>0$. Choose $\delta \equiv \delta(N)$ such that $N \delta \rightarrow \infty$ and $N \delta^{2} \rightarrow 0$; we may for instance adopt $\delta=N^{-3 / 5}$. We have, by monotonicity of the integrand:

$$
J_{N}(\xi)=\int_{0}^{\delta}(1-y)^{\xi N}(1+y)^{(1-\xi) N} \frac{d y}{1-y^{2}}(1+\mathcal{E} \mathcal{S}(N)) .
$$

The integrand is of the form $e^{N g(y)}$, where the function

$$
g(y):=\xi \log (1-y)+(1-\xi) \log (1+y)
$$

satisfies $g(y)=(1-2 \xi) y+O\left(y^{2}\right)$ for small $y$. Thus, locally expanding then completing the tails yields:

$$
\begin{aligned}
J_{N}(\xi) & =\int_{0}^{\delta} e^{-N(2 \xi-1) y} d y\left(1+O\left(N \delta^{2}\right)\right) \\
& =\int_{0}^{\infty} e^{-N(2 \xi-1) y} d y\left(1+O\left(N^{-1 / 5}\right)\right) \\
& =\frac{1}{N(2 \xi-1)}\left(1+O\left(N^{-1 / 5}\right)\right) .
\end{aligned}
$$

It then proves safe to replace $\xi$ by $x$, since error terms can be checked to be uniform with respect to $\xi$, once $\xi$ is confined to a small interval excluding $\frac{1}{2}$ (we shall freely do so in what follows). We have thus found that

$$
\omega_{N, k}=2^{-N+1}\left(\begin{array}{c}
N-2 \\
k-1
\end{array}\right) \frac{1}{2 x-1}(1+o(1)) .
$$

The stated estimate 190 results from here by the usual asymptotic approximation of the binomial coefficients $\left(\begin{array}{c}N \\ k\end{array}\right)$, whenever $N$ and $k$ are proportional. (Stirling's formula leads classically and elementarily to the entropy function.)

Note 2. (i) The expressions degenerate, as they should, as $x$ approaches $\frac{1}{2}$.

(ii) Let $\widetilde{\omega}_{N, k}$ be the rough approximation 190 of the statement, namely,

$$
\widetilde{\omega}_{N, k}:=\exp (-N(\log 2+x \log (x)+(1-x) \log (1-x))), \quad x=\frac{k}{N},
$$


and $\omega_{N, k}^{\star}$ the approximation of $[22]$, namely,

$$
\omega_{N, k}^{\star}:=2^{-N+1}\left(\begin{array}{c}
N-2 \\
k-1
\end{array}\right) \frac{1}{2 x-1}, \quad x=\frac{k}{N} .
$$

Here are typical numerical data, obtained for $N=10^{4}$ :

\begin{tabular}{l|cccc}
\hline$x$ & 0.6 & 0.7 & 0.8 & 0.9 \\
\hline$\omega_{N, k}$ & $6.958 \cdot 10^{-90}$ & $4.080 \cdot 10^{-360}$ & $4.424 \cdot 10^{-840}$ & $9.847 \cdot 10^{-1602}$ \\
$\widetilde{\omega}_{N, k}$ & $3.569 \cdot 10^{-88}$ & $4.466 \cdot 10^{-358}$ & $8.320 \cdot 10^{-838}$ & $3.291 \cdot 10^{-1559}$ \\
$\omega_{N, k}^{\star}$ & $6.976 \cdot 10^{-90}$ & $4.083 \cdot 10^{-360}$ & $4.426 \cdot 10^{-840}$ & $9.850 \cdot 10^{-1602}$. \\
\hline
\end{tabular}

The discrepancy for $\widetilde{\omega}_{N, k}$, corresponds to a comparatively small factor that, in accordance with previous calculations, is of polynomial growth in $N$. The binomial approximation $\omega_{N, k}^{\star}$ is extremely good. The merit of $\widetilde{\omega}_{N, k}$ is to provide a simple-to-use formula that predicts the right order of the probabilities involved.

(iii) For the French sheep system evoked in the introduction $\left(N=6 \cdot 10^{7}\right)$, the probability of winning against a majority of $53 \%, 51 \%$, and $50.1 \%$ are estimated by 22 to be respectively about $2 \cdot 10^{-46,935}, 8 \cdot 10^{-5,215}$, and $4 \cdot 10^{-54}$.

\subsection{Time till absorption}

The analysis of the limit law of extinction time, in the unfair case $x<\frac{1}{2}$, again involves Laplace's method, albeit with a different geometry. The starting point is the integral representation 17 of the probability generating function $\phi_{N, k}(u)$. When $u=1$ and $x<\frac{1}{2}$, there is a unique maximum of the integrand, but it is now located at an interior point of $(0,1)$. As in Subsection 4.1, only a small interval around this maximum matters; one can then approximate the integrand by the exponential of a quadratic form. Next, when $u=e^{i \tau}$, it proves possible to develop a perturbative analysis and estimate the value of $\phi_{N, k}\left(e^{i \tau}\right)$. With a suitable normalization, namely, $\tau=t / \sqrt{N}$ with $t$ fixed, one gains access in this way to the characteristic function of the (properly scaled) $A$-extinction time $T$. Convergence to the characteristic function $e^{-t^{2} / 2}$ of a standard normal variable is then sufficient to conclude. We carry out this programme in the proof of the following statement:

Theorem 3 (Unfair urn: Gaussian limit law) Consider the Mabinogion urn $\mathcal{M}[N]$ with initial composition $\left(A_{0}, B_{0}\right)=(k, N-k)$ and $k=\lfloor x N\rfloor$, where $0<x<\frac{1}{2}$. The A-extinction time $T$ converges in distribution to a normal: for all $t \in \mathbb{R}$, one has

$$
\lim _{N \rightarrow \infty} \mathbb{P}\left(\frac{T-\tau(x) N}{\sigma(x) \sqrt{N}} \leq t\right)=\frac{1}{\sqrt{2 \pi}} \int_{-\infty}^{t} e^{-w^{2} / 2} d w
$$

where the normalizing quantities $\sigma(x), \tau(x)$ are

$$
\tau(x):=\frac{1}{2} \log \frac{1}{1-2 x}, \quad \sigma(x)^{2}=\frac{1}{2} \log (1-2 x)+\frac{x(1-x)}{(1-2 x)^{2}}
$$


Proof: The change of variable $y=e^{-2 z u / N}$ in (17) yields an integral representation of $\phi_{N, k}(u)$, which is valid for $u \in(0,1)$ and, by analytic continuation, to larger regions of the complex $u$-plane:

$$
\left\{\begin{aligned}
\phi_{N, k}(u) & =\frac{N-1}{2^{N-1}}\left(\begin{array}{c}
N-2 \\
k-1
\end{array}\right) J_{n}(x, u) \\
J_{N}(x, u) & :=\int_{0}^{1} y^{N(1 / u-1) / 2}(1-y)^{x N}(1+y)^{(1-x) N} \frac{d y}{1-y^{2}} .
\end{aligned}\right.
$$

(We consider directly the case $k / N=x$.) We propose to estimate the characteristic function

$$
\mathbb{E}\left[e^{i t T / \sqrt{N}}\right]=\phi_{N, k}\left(e^{i t / \sqrt{N}}\right),
$$

for an arbitrary fixed $t \in \mathbb{R}$; this forces the quantity $u=e^{i t / \sqrt{N}}$ in the PGF to be close to 1 .

First, we rewrite the main integral as

$$
J_{N}(x, u)=\int_{0}^{1} y^{N(1 / u-1) / 2} e^{N g(y)} \frac{d y}{1-y^{2}},
$$

where the function $g(y)$ is

$$
g(y)=x \log (1-y)+(1-x) \log (1+y) .
$$

A calculation of the derivative shows that $g(y)$ is unimodal, with a maximum at

$$
y_{0}=1-2 x,
$$

which, for $x<\frac{1}{2}$, is interior to the interval $(0,1)$.

For $u=1$, we have (with the notation 21) )

$$
J_{N}(x, 1)=\int_{y_{0}-\delta}^{y_{0}+\delta} e^{N g(y)} \frac{d y}{1-y^{2}}+e^{N g\left(y_{0}\right)} \mathcal{E} \mathcal{S}(N),
$$

which, by unimodality, holds for fixed $\delta$ and remains valid as long as $N \delta^{2} \rightarrow \infty$ sufficiently rapidly. Imposing next $N \delta^{3} \rightarrow 0$ makes it possible to reduce $g(y)$ to its quadratic expansion near $y_{0}$. Accordingly, we choose $\delta=N^{-2 / 5}$, which satisfies both previous requirements. We have, for $\left|y-y_{0}\right|<\delta$,

$$
e^{N g(y)}=e^{N g\left(y_{0}\right)+N\left(y-y_{0}\right)^{2} g^{\prime \prime}\left(y_{0}\right) / 2}\left(1+O\left(N^{-1 / 5}\right)\right),
$$

where $g^{\prime \prime}\left(y_{0}\right)=-(4 x(1-x))^{-1}$. Setting $y-y_{0}=w / \sqrt{N}$ and completing the tails then provides the asymptotic form

$$
J_{N}(x, 1)=\frac{\sqrt{2 \pi}}{\sqrt{-N g^{\prime \prime}\left(y_{0}\right)}} \frac{e^{N g\left(y_{0}\right)}}{1-y_{0}^{2}}\left(1+O\left(N^{-1 / 5}\right)\right) .
$$

Observe finally that, when $u=1$, Theorem 1 implies the estimate $\phi_{N, k}(1)=1-o(1)$ (in fact, the error term $o(1)$ is exponentially small by Theorem 2). Thus, we also have, without new computations:

$$
\frac{N-1}{2^{N-1}}\left(\begin{array}{c}
N-2 \\
k-1
\end{array}\right) \frac{\sqrt{2 \pi}}{\sqrt{-N g^{\prime \prime}\left(y_{0}\right)}} \frac{e^{N g\left(y_{0}\right)}}{1-y_{0}^{2}}=\left(1+O\left(N^{-1 / 5}\right)\right) \text {. }
$$


We can now proceed with the case $u=e^{i t / \sqrt{N}}$ and adopt the same split of the integration domain into the small interval $\left[y_{0}-\delta, y_{0}+\delta\right]$ and its complement, with (still) $\delta=N^{-2 / 5}$. For $y$ restricted to $\left[y_{0}-\delta, y_{0}+\delta\right]$, the powers of $y$ are estimated by

$$
\begin{aligned}
y^{N\left(u^{-1}-1\right) / 2} & =\exp \left(\frac{N}{2}\left(e^{-i t / \sqrt{N}}-1\right) \log y\right) \\
& =y^{-i t \sqrt{N} / 2} y^{-t^{2} / 4}\left(1+O\left(N^{-1 / 2}\right)\right)
\end{aligned}
$$

as follows from a three-term expansion of $e^{i t / \sqrt{N}}$. In the second line of $(29)$, the first factor is of modulus equal to 1 , no matter what the values of $y \in(0,1)$ and $t \in \mathbb{R}$ are. The second one remains $O(1)$. Thus, proceeding as in 26), we obtain

$$
J_{N}\left(x, e^{i t / \sqrt{N}}\right)=\int_{y_{0}-\delta}^{y_{0}+\delta} y^{-i t \sqrt{N} / 2} y^{-t^{2} / 4}\left(1+O\left(N^{-1 / 2}\right)\right) e^{N g(y)} \frac{d y}{1-y^{2}}+e^{N g\left(y_{0}\right)} \mathcal{E} \mathcal{S}(N) .
$$

Making use of 27] and 29] gives

$$
\begin{gathered}
J_{N}\left(x, e^{i t / \sqrt{N}}\right)=\frac{e^{N g\left(y_{0}\right)}}{1-y_{0}^{2}} \int_{y_{0}-\delta}^{y_{0}+\delta} y^{-i t \sqrt{N} / 2} y^{-t^{2} / 4} e^{N\left(y-y_{0}\right)^{2} g^{\prime \prime}\left(y_{0}\right) / 2}\left(1+\epsilon_{N}\right) \frac{d y}{1-y^{2}} \\
+e^{N g\left(y_{0}\right)} \mathcal{E} \mathcal{S}(N),
\end{gathered}
$$

where $\epsilon_{N}=O\left(N^{-1 / 5}\right)$.

We are now ready for the final move. Set $y-y_{0}=w / \sqrt{N}$, which implies that $w$ varies in the interval $\left[-N^{-1 / 10}, N^{-1 / 10}\right]$. We only need to expand again the integrand of 30 (with some care) and collect the main terms:

$$
y^{-t^{2} / 4-i t \sqrt{N} / 2}=y_{0}^{-t^{2} / 4-i t \sqrt{N} / 2} \exp \left(-\frac{i t w}{2 y_{0}}\right)\left(1+O\left(N^{-1 / 10}\right)\right) .
$$

Inserting this last approximation into 30 and completing the tails then yields

$$
\begin{aligned}
& J_{N}\left(x, e^{i t / \sqrt{N}}\right) \sim {\left[\frac{e^{N g\left(y_{0}\right)} y_{0}^{-t^{2} / 4-i t \sqrt{N} / 2}}{\left(1-y_{0}^{2}\right) \sqrt{N}}\right] } \\
& \cdot \int_{-\infty}^{+\infty} \exp \left(-\frac{i t w}{2 y_{0}}+\frac{w^{2}}{2} g^{\prime \prime}\left(y_{0}\right)\right) d w .
\end{aligned}
$$

The integral can be evaluated by shifting vertically the integration line and making use of Cauchy's Theorem. To wit:

$$
\int_{-\infty}^{+\infty} e^{-\gamma w^{2} / 2+i \xi w} d w=e^{-\xi^{2} /(2 \gamma)} \sqrt{\frac{2 \pi}{\gamma}} \quad(\gamma>0) .
$$

In summary, from 25, 28, 231, and 32, we find:

$$
\phi_{N, k}\left(e^{i t / \sqrt{N}}\right) \sim \exp (i t \sqrt{N} \tau(x)) \cdot \exp \left(-\frac{t^{2}}{2}\left[\frac{1}{2} \log y_{0}+\frac{1}{4 y_{0}^{2}\left|g^{\prime \prime}\left(y_{0}\right)\right|}\right]\right) .
$$



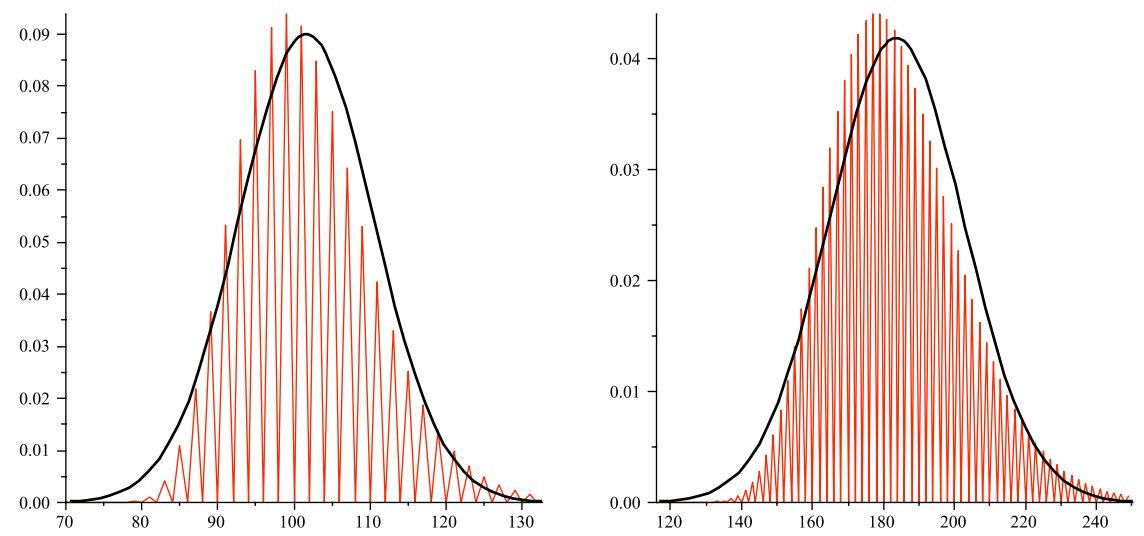

Fig. 3: The histograms of the distribution of the $A$-extinction time in the case $N=400$, for $k=80, x=0.2$ [left] and $k=120, x=0.3$ [right], compared to a Gaussian density with matching mean and variance.

This last estimate is enough to guarantee that the random quantity (with $\tau$ as in (24))

$$
T^{\star}:=\frac{T-N \tau(x)}{\sqrt{N}},
$$

has its characteristic function satisfying

$$
\mathbb{E}\left(e^{i t T^{\star}}\right) \sim \exp \left(-\frac{t^{2}}{2}\left[\frac{1}{2} \log (1-2 x)+\frac{x(1-x)}{(1-2 x)^{2}}\right]\right) .
$$

The continuity theorem for characteristic functions [2], then implies that $T^{\star}$ is asymptotically normally distributed. The proof is now complete.

Note 3. ( $i$ ) The speed of convergence to the Gaussian limit, as measured by the distance betwen the distribution functions in (23) can be bounded from above thanks to the Berry-Esseen inequalities [28]: our proof implies a speed of $O\left(N^{-1 / 1 \sigma}\right)$. It is probably the case that, by pushing all expansions one step further, one could obtain the actual speed as being about $O\left(N^{-1 / 2}\right)$.

(ii) Numerical calculations indicate that a local limit law holds: see Figure 3 In other words, the individual probabilities $\mathbb{P}(T=N \tau(x)+\lambda \sigma(x) \sqrt{N})$ are asymptotically expressible in terms of the Gaussian density $e^{-\lambda^{2} / 2} / \sqrt{2 \pi}$ : histograms converge to the famous bell-shaped curve. A plausible path to the proof is the saddle point method applied to Cauchy coefficient integrals, in the style of what is done in the next section for the fair case.

(iii) Moments can be obtained by differentiating under the integral sign in 25 , then setting $u=1$. What results are integral representations akin to the ones treated in the previous subsection (with extra polynomial factors in $z$ now entering the integrand). Laplace's method is again instrumental in analysing these. For instance, we find in this way that $\mathbb{E}(T) \sim \tau(x) N, \mathbb{E}\left(T^{2}\right) \sim \tau(x)^{2} N^{2}, \mathbb{V}(T) \sim \sigma(x)^{2} N$, and so on. Thus, the constants $\tau, \sigma$ are not just normalizing constants for the Gaussian limit law; they also occur in the asymptotic form of the moments. 


\section{The fair Mabinogion urn}

We now analyse the case of a fair urn, which has a total population of even size, $N=2 \nu$, and is initialized with an equal number of balls of each type: $\left(A_{0}, B_{0}\right)=(\nu, \nu)$. The random variable of interest is now the random variable $\widehat{T}$, which represents the "absolute" time till absorption, corresponding to extinction of either the $A$-balls or the $B$-balls. The quantity $\widehat{T}$ is finite with probability 1 (contrary to the time $T$ till $A$-extinction, considered previously, which is a defective random variable). In Subsection 5.1, we characterize the distribution of $\widehat{T}$ as a sum of geometrics with quadratically varying rates (Proposition 1 ). In Subsection 5.2, we establish the existence of a limit distribution, which is of a double-exponential type and prove a local limit theorem (Theorem 47) this limit law can also be described as a sum with harmonic weights of exponential variables.

\subsection{Exact distribution of the absolute extinction time.}

By the symmetry inherent in the fair case, we have

$$
\widehat{\phi}_{2 \nu, \nu}(u):=\mathbb{E}\left(e^{u \widehat{T}}\right)=2 \phi_{2 \nu, \nu}(u)
$$

As we are going to see the distribution of $\widehat{T}$ admits of a surprising form. A random variable $X$ is said to be geometric with rate $\lambda$ if

$$
\mathbb{P}(X=k)=\frac{\lambda^{k}}{1-\lambda}, \quad k \in \mathbb{Z}_{\geq 0}
$$

The PGF of such a variable is

$$
\mathbb{E}\left(u^{X}\right) \equiv \sum_{k \geq 0} \mathbb{P}(X=k) u^{k}=\frac{1-\lambda}{1-\lambda u}
$$

it has mean $\mathbb{E}(X)=\lambda /(1-\lambda)$ and variance $\mathbb{V}(X)=\lambda /(1-\lambda)^{2}$. We state:

Proposition 1 In the fair case $N=2 \nu$ even and an initial composition $\left(A_{0}, B_{0}\right)=(\nu, \nu)$, the PGF of the absolute extinction time $\widehat{T}$ is

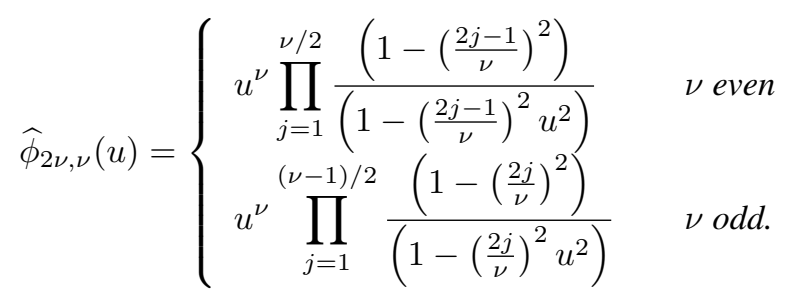

Equivalently, with $\mathbf{G}[\lambda]$ representing a geometric random variable of rate $\lambda$, the following distributional equivalence holds (with independence of the various geometric variables involved):

$$
\widehat{T} \stackrel{\mathrm{d}}{=}\left\{\begin{array}{cc}
\nu+2 \sum_{j=1}^{\nu / 2} \mathbf{G}\left[\left(\frac{2 j-1}{\nu}\right)^{2}\right] & \nu \text { even } \\
\nu+2 \sum_{j=1}^{(\nu-1) / 2} \mathbf{G}\left[\left(\frac{2 j}{\nu}\right)^{2}\right] & \nu \text { odd } .
\end{array}\right.
$$


Proof: The generating function of $\widehat{T}$ is obtained by setting $N=2 \nu$ and $k=N-k=\nu$ in (25), then making use of 33 :

$$
\widehat{\phi}_{2 \nu, \nu}(u)=\frac{2 \nu-1}{2^{2 \nu-2}}\left(\begin{array}{c}
2 \nu-2 \\
\nu-1
\end{array}\right) \int_{0}^{1} y^{\nu\left(u^{-1}-1\right)}\left(1-y^{2}\right)^{\nu-1} d y .
$$

The change of variable $Y=y^{2}$ reduces the integral to an Eulerian Beta function [37],

$$
B(\alpha, \beta):=\int_{0}^{1} t^{\alpha-1}(1-t)^{\beta-1} d t=\frac{\Gamma(\alpha) \Gamma(\beta)}{\Gamma(\alpha+\beta)},
$$

so that

$$
\begin{aligned}
\widehat{\phi}_{2 \nu, \nu}(u) & =\frac{2 \nu-1}{2^{2 \nu-1}}\left(\begin{array}{c}
2 \nu-2 \\
\nu-1
\end{array}\right) B\left(\frac{\nu}{2}\left(u^{-1}-1\right)+\frac{1}{2}, \nu\right) \\
& =\frac{2 \nu-1}{2^{2 \nu-1}}\left(\begin{array}{c}
2 \nu-2 \\
\nu-1
\end{array}\right) \frac{\Gamma\left(-\frac{(\nu-1)}{2}+\frac{\nu}{2 u}\right) \Gamma(\nu)}{\Gamma\left(\frac{\nu+1}{2}+\frac{\nu}{2 u}\right)} .
\end{aligned}
$$

The basic functional property of the Gamma function, $\Gamma(s+1)=s \Gamma(s)$, then gives us a rational form

$$
\begin{aligned}
\widehat{\phi}_{2 \nu, \nu}(u) & =\frac{2 \nu-1}{2^{2 \nu-1}} \frac{(2 \nu-2) ! 2^{\nu} u^{\nu}}{(\nu-1) !} \prod_{\ell=1}^{\nu}(\nu-u(\nu+1-2 \ell))^{-1} \\
& =u^{\nu} \prod_{\ell=1}^{\nu} \frac{1-a_{\ell}}{1-u a_{\ell}},
\end{aligned}
$$

where $a_{\ell}=(2 \ell-\nu-1) / \nu$ varies in the interval $-\{(\nu-1) / \nu,(\nu-1) / \nu\} \subset[-1,1]$. The terms corresponding to values of $a_{\ell}$ that are symmetric around 0 can then be grouped together. This eventually leads us to distinguish two cases, depending upon the parity of $\nu$, and the statement follows by simple algebra.

Note 4. It is a general theorem ${ }^{[\text {(iii) }}$ that, for a discrete-time birth-and-death chain on the state space $\{0, \ldots, d\}$, started at 0 and having $d$ as an absorbing state, the absorption time has a probability generating function of the form

$$
u^{d} \prod_{j=0}^{d-1} \frac{1-\theta_{j}}{1-\theta_{j} u},
$$

where the $\theta_{j}$ are real numbers of the interval $(-1,1)$ that are related to spectral properties of the chain's kernel. (Thus, the absorption time is distributed as a sum of "geometric" random variables, provided we allow "geometric" random variables of negative rates.) This theorem is enunciated and proved by Fill in [10 Th. 2], who observes that it constitutes the discrete-time analogue of a classical theorem relative to continuous-time processes and usually attributed to Keilson (see [23, §1] and Theorem 5.1A of [24].) Our Proposition 1] can thus be regarded as a particular incarnation of the Keilson-Fill Theorem.

We also observe that the Keilson-Fill Theorem is itself logically equivalent to the enumeration of weighted lattice paths that cross a strip: by a general result of Flajolet [11 §3.1], the corresponding generating function is invariably proportional to the inverse of a polynomial. In the case of the Ehrenfest-Mabinogion urn, the polynomials are none other than the denominators of the convergents of the continued fraction 14.

(iii) We are grateful to Jim Fill (private communication, April 2008) for these remarks. 


\subsection{Limit distribution.}

Based on the formulae of Proposition 1, it is now a simple task to derive explicit expressions for moments of the absolute extinction time $\widehat{T}$. The expressions involve either the usual $\psi$ function [37, p. 241]

$$
\psi(s):=\frac{d}{d s} \log \Gamma(s)=-\gamma-\frac{1}{s}+\sum_{m=1}^{\infty}\left(\frac{1}{m}-\frac{1}{m+s}\right),
$$

or the generalized harmonic numbers

$$
H_{n}^{(a)}=\sum_{m=1}^{n} \frac{1}{m^{a}}
$$

for (small) integral values of the parameter $a$. In particular, $H_{n} \equiv H_{n}^{(1)}$ is the usual harmonic number. We have:

Proposition 2 In the fair case, $N=2 \nu$, the mean and variance of the absolute absorption time $\widehat{T}$ satisfy:

$$
\begin{aligned}
\mathbb{E}(\widehat{T}) & =\frac{\nu}{2}\left(\psi\left(\nu+\frac{1}{2}\right)+2 \log 2+\gamma\right) \\
& =\nu\left[H_{2 \nu}-\frac{1}{2} H_{\nu}\right] \\
& \sim \frac{\nu}{\nu \rightarrow \infty}(\log \nu+2 \log 2+\gamma)+\frac{1}{48 \nu}+O\left(\frac{1}{\nu^{3}}\right) \\
\mathbb{V}(\widehat{T})= & \frac{\pi^{2} \nu^{2}}{8}-\frac{\nu}{2}\left(\psi\left(\nu+\frac{1}{2}\right)+2 \log 2+\gamma\right)-\frac{\nu^{2}}{4} \psi^{\prime}\left(\nu+\frac{1}{2}\right) \\
& =\frac{\nu^{2}\left[H_{2 \nu}^{(2)}-\frac{1}{4} H_{\nu}^{(2)}\right]-\nu\left[H_{2 \nu}-\frac{1}{2} H_{\nu}\right]}{8} \\
& \frac{\pi^{2} \nu^{2}}{8}-\frac{\nu}{2}\left(\log \nu+2 \log 2+\gamma+\frac{1}{2}\right)+O\left(\frac{1}{\nu^{3}}\right) .
\end{aligned}
$$

Thus, the mean is asymptotic to $\frac{1}{2} \nu \log \nu$ and the standard deviation is of the order of $\nu$. This information makes it possible to determine the region where most of the probability mass is concentrated, and then, after a proper scaling, determine the shape of the limit law of absorption time.

Theorem 4 (Fair urn: local limit) Consider the Mabinogion urn $\mathcal{M}[2 \nu]$ in the fair case $\left(A_{0}, B_{0}\right)=$ $(\nu, \nu)$. Let $\nu, n \rightarrow \infty$ in such a way that

$$
n=\frac{1}{2} \nu \log \nu+t \nu
$$

Then, the absolute extinction time $\widehat{T}$ satisfies, for $t$ in a compact set of $\mathbb{R}$ and $n-\nu$ even,

$$
\mathbb{P}(\widehat{T}=n+1) \sim \frac{2}{\nu} \cdot C e^{-t} e^{-e^{-2 t}},
$$

with $C=2 / \sqrt{\pi}$. 
Proof: For the sake of notational simplicity, we shall analyse the urn of size $N=2 \nu+2$, in the fair case $\left(A_{0}, B_{0}\right)=(\nu+1, \nu+1)$, the translation $\nu \mapsto \nu-1$ being then an easy task. The starting point is the formula (3) of Theorem 1], which gives, relative to $\mathcal{M}[2 \nu+2]$ with $k=\nu+1$ :

$$
\mathbb{P}(\widehat{T}=n+1)=2 \cdot \frac{2 \nu+1}{2 \nu+2} \frac{n !}{(\nu+1)^{n} 2^{\nu}}\left(\begin{array}{c}
2 \nu \\
\nu
\end{array}\right)\left[z^{n}\right](\sinh z)^{\nu} .
$$

(The prefactor 2 takes care of the symmetry due to extinction of either the As or the Bs; also the duplication formula for hyperbolic sine has been used.) What is needed is thus an estimation of coefficients of large index $(n)$ in a large power $(\nu)$ of the fixed function $\sinh z$, in the regime $n \sim \frac{1}{2} \nu \log \nu$. This problem is approached by means of Cauchy's coefficient formula

$$
\left[z^{n}\right](\sinh z)^{\nu}=\frac{1}{2 i \pi} \int_{\gamma} \frac{(\sinh z)^{\nu}}{z^{n}} \frac{d z}{z}
$$

where we choose $\gamma$ to be a circle centred at the origin of a yet unspecified positive radius.

We henceforth impose that $n$ is close enough to $\frac{1}{2} \nu \log \nu$ and postulate at this stage the following crude conditions:

$$
\frac{2}{5} \log \nu \leq \frac{n}{\nu} \leq \frac{3}{5} \log \nu
$$

Also, since $n-\nu$ is assumed to be even, we have, by symmetry,

$$
\left[z^{n}\right](\sinh z)^{\nu}=\frac{1}{i \pi} \int_{\gamma^{+}} \frac{(\sinh z)^{\nu}}{z^{n}} \frac{d z}{z},
$$

where $\gamma^{+}$is the subset of $\gamma$ contained in the half-plane $\Re(z) \geq 0$. Since $n$ and $\nu$ are not proportional, the usual treatment of large coefficients in large powers (as in [13, §VIII.8]) is not applicable. Nonetheless, it is still natural to opt for a saddle-point analysis, following the general principles of this classical method [4, [13, 29]. Accordingly, we shall integrate along a circle of radius $\lambda$ such that

$$
\left.\frac{d}{d z} \frac{(\sinh z)^{\nu}}{z^{n}}\right|_{z=\lambda}=0 ; \quad \text { that is: } \quad \lambda \operatorname{coth} \lambda=\frac{n}{\nu} .
$$

Given the range condition 40 , we have $\lambda \rightarrow+\infty$, and indeed

$$
\lambda\left(1+2 e^{-2 \lambda}+O\left(e^{-4 \lambda}\right)\right)=\frac{n}{\nu},
$$

which implies that $\lambda \sim n / \nu$; in particular, $\lambda=O(\log \nu)$ and $\lambda=O(\log n)$.

It will prove convenient to set

$$
K_{\nu, n}(\varphi):=\int_{\gamma(\varphi)} \frac{(\sinh z)^{\nu}}{z^{n}} \frac{d z}{z}, \quad \text { where } \quad \gamma(\varphi)=\left\{\lambda e^{i \theta} \mid-\varphi \leq \theta \leq \varphi\right\} .
$$

Elementary trigonometry yields

$$
\left|\sinh \left(\lambda e^{i \theta}\right)\right|^{2}=\cosh ^{2}(\lambda \cos \theta)-\cos ^{2}(\lambda \sin \theta) .
$$


Thus, for $\nu$ large enough, one has

$$
K_{\nu, n}(\pi / 2)-K_{\nu, n}(\pi / 4)=O\left(\left(0.51 e^{\lambda / \sqrt{2}}\right)^{\nu} \lambda^{-n}\right)
$$

which is exponentially small compared to the value of the integrand at $\lambda$; namely, $(\sinh \lambda)^{\nu} \lambda^{-n}$.

Next, we fix a small angle

$$
\theta_{0}=n^{-2 / 5}
$$

chosen such that $n \theta_{0}^{2} \rightarrow \infty$ and $n \theta_{0}^{3} \rightarrow 0$. Trivial bound combined with the relation (44) provide the estimate

$$
K_{\nu, n}(\pi / 4)-K_{\nu, n}\left(\theta_{0}\right)=O\left(\cosh ^{\nu}\left(\lambda \cos \theta_{0}\right) \lambda^{-n}\right)=(\sinh \lambda)^{\nu} \lambda^{-n} \mathcal{E} \mathcal{S}(n),
$$

where the notation $\sqrt{21}$ for exponentially small terms is employed.

From (45) and 46), we thus expect that only the contribution along the small circular arc $\gamma\left(\theta_{0}\right)$ should matter. There, as it is typically the case, local expansions suffice to estimate the integral. We then define

$$
h(z)=\nu \log \sinh z-n \log z
$$

so that

$$
h^{\prime}(z)=\nu \operatorname{coth} z-\frac{n}{z}, \quad h^{\prime \prime}(z)=-\frac{\nu}{\sinh ^{2} z}+\frac{n}{z^{2}},
$$

and, as we know, $\lambda$ is defined by $h^{\prime}(\lambda)=0$. In addition, it is easily verified that $h^{\prime \prime \prime}(z)=O\left(n / \lambda^{3}\right)$ on $\gamma\left(\theta_{0}\right)$. Consequently, for $z \in \gamma\left(\theta_{0}\right)$,

$$
h(z)=h(\lambda)+\frac{1}{2} h^{\prime \prime}(\lambda)(z-\lambda)^{2}+O\left(n \theta_{0}^{3}\right)
$$

and the error term is $O\left(n^{-1 / 5}\right)$.

By analogy with $(21)$, we let $\mathcal{P} \mathcal{S}(n)$ denote any quantity that is polynomially small in $n$, in the sense that

$$
f(n)=\mathcal{P} \mathcal{S}(n) \quad \text { iff } \quad f(n)=O\left(n^{-L}\right),
$$

for some $L>0$. We then have, from 47 :

$$
\begin{aligned}
K_{\nu, n}\left(\theta_{0}\right) & =e^{h(\lambda)} \int_{\gamma\left(\theta_{0}\right)} \exp \left(\frac{1}{2} h^{\prime \prime}(\lambda)(z-\lambda)^{2}\right)(1+\mathcal{P} \mathcal{S}(n)) \frac{d z}{z} \\
& =\frac{\sinh ^{\nu} \lambda}{\lambda^{n+1}} \int_{\gamma\left(\theta_{0}\right)} \exp \left(\frac{1}{2} h^{\prime \prime}(\lambda)(z-\lambda)^{2}\right)(1+\mathcal{P} \mathcal{S}(n)) d z
\end{aligned}
$$

The integral in 49 is now estimated by setting $z=\lambda+i t$, and then completing the integration range to $t \in(-\infty,+\infty)$; this at the expense of exponentially small error terms. In this way, we obtain

$$
\begin{aligned}
\int_{\gamma\left(\theta_{0}\right)} \exp \left(\frac{1}{2} h^{\prime \prime}(\lambda)(z-\lambda)^{2}\right) d z & =i \int_{-\infty}^{+\infty} \exp \left(-\frac{1}{2} h^{\prime \prime}(\lambda) t^{2}\right) d t(1+\mathcal{E} \mathcal{S}(n)) \\
& =i \sqrt{\frac{2 \pi}{h^{\prime \prime}(\lambda)}}(1+\mathcal{E} \mathcal{S}(n)) .
\end{aligned}
$$




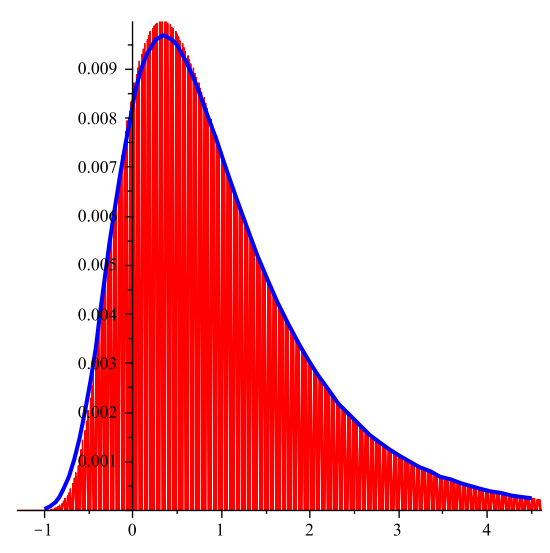

Fig. 4: The histogram of the distribution of the absolute extinction time $\widehat{T}_{\nu}^{\star}$ of a fair urn, normalized according to 54) for $N=2 \nu=200$ [polygonal line] and compared to the limit density [continuous curve] scaled according to Eq. (37).

Then, 49 and 50 combined yield

$$
K_{\nu, n}\left(\theta_{0}\right)=\frac{\sinh ^{\nu} \lambda}{\lambda^{n}} \sqrt{\frac{2}{\pi n}}(1+\mathcal{P} \mathcal{S}(n)) .
$$

We can now complete the proof: by $(38,4), 45,446$, and $(51)$, we find:

$$
\mathbb{P}(\widehat{T}=n+1)=2 \frac{n !}{(\nu+1)^{n} 2^{\nu}}\left(\begin{array}{c}
2 \nu \\
\nu
\end{array}\right) \frac{\sinh ^{\nu} \lambda}{\lambda^{n}} \sqrt{\frac{2}{\pi n}}(1+\mathcal{P} \mathcal{S}(n)) .
$$

It is then a routine (but tedious) matter to verify the statement from (52), using the basic relations

$$
\left\{\begin{array}{l}
n=\frac{1}{2} \nu \log \nu+t \nu \\
\lambda\left(1+2 e^{-2 \lambda}+O\left(e^{-4 \lambda}\right)\right)=\frac{n}{\nu},
\end{array}\right.
$$

as well as standard asymptotic expansions like

$$
\log \sinh z=z-\log 2-e^{-2 z}+O\left(e^{-4 z}\right)
$$

and Stirling's formula.

Figure 4 shows the excellent agreement between the exact probabilities and the continuous limit, even for a value of $\nu$ as low as 100 .

Note 5. (i) By summation of individual probabilities, the local limit law expressed by the statement implies convergence in distribution of the normalized version of $\widehat{T} \equiv \widehat{T}_{\nu}$,

$$
\frac{1}{\nu}\left(\widehat{T}_{\nu}-\frac{1}{2} \nu \log \nu\right) \stackrel{\mathrm{d}}{\rightarrow} \widehat{T}_{\infty}
$$


where $\widehat{T}_{\infty}$ has the density $C e^{-t} e^{-e^{-2 t}}$ expressed by 37 . (The factor 2 in 37 corresponds to the fact that only half of the probabilities are nonzero, due to parity constraints.) As is apparent from the shape of this density, the limit law is of an "exp-gamma(1/2)" type, in the sense that $\widehat{T}_{\infty}=-\frac{1}{2} \log X$, where $X$ is gamma(1/2) distributed:

$$
\mathbb{P}(X \in(x, x+d x))=\frac{1}{\sqrt{\pi}} e^{-x} x^{-1 / 2} d x .
$$

(ii) The weaker convergence-in-distribution result 53 can be directly derived from Proposition 2 by means of characteristic functions. Indeed, by setting $u=e^{i t / \nu}$ in $\phi_{2 \nu, \nu}(u)$ as given by (34) and normalizing, we find that the random variable

$$
\widehat{T}_{\nu}^{\star}:=\frac{1}{\nu}\left(\widehat{T}_{\nu}-\frac{1}{2} \nu \log \nu\right)
$$

has its characteristic function that satisfies

$$
\mathbb{E}\left(e^{i t \widehat{T}_{\nu}^{\star}}\right)=\nu^{-i t / 2} \widehat{\phi}_{2 \nu, \nu}\left(e^{i t / \nu}\right) \underset{\nu \rightarrow \infty}{\sim} \nu^{-i t / 2} \prod_{\ell=1}^{\nu} \frac{2 \ell-1}{2 \ell-1-i t} .
$$

On the other hand, a classical limit formula for the Gamma function is

$$
\Gamma(s)=\lim _{\nu \rightarrow \infty} \frac{\nu ! \nu^{s}}{s(s+1) \cdots(s+\nu)}, \quad s \in \mathbb{C} \backslash \mathbb{Z}_{\leq 0}
$$

A comparison of 55 and 56 then shows that

$$
\lim _{\nu \rightarrow \infty} \mathbb{E}\left(e^{i t \widehat{T}_{\nu}^{\star}}\right)=\Phi(t), \quad \text { where } \quad \Phi(t)=\frac{\Gamma\left(\frac{1-i t}{2}\right)}{\Gamma\left(\frac{1}{2}\right)} .
$$

Thus, by the continuity theorem for characteristic functions, the normalized random variable $\widehat{T}_{\nu}^{\star}$ converges in distribution to a random variable, which admits $\Phi(t)$ as its characteristic function. (It can be verified independently that $\Phi(t)$ is the characteristic function of a variable whose density is $C e^{-t} e^{-e^{-2 t}}$.)

(iii) From the developments of Part (ii), especially Eq. (55), there also results that $\widehat{T}_{\infty}$ is characterized in distribution as the limit of a weighted sum of independent identically distributed random variables $E_{\ell}$,

$$
\sum_{\ell=1}^{\nu} \frac{1}{2 \ell-1} E_{\ell}-\frac{1}{2} \log \nu \stackrel{\mathrm{d}}{\rightarrow} \widehat{T}_{\infty}
$$

where the $E_{\ell}$ are exponential of rate 1 ; that is, $\mathbb{P}\left(E_{\ell} \leq x\right)=1-e^{-x}$. This last expression implies that $\widehat{T}_{\infty}$ has a property known as self-decomposability ${ }^{\text {(iv) }}$, which means that $\widehat{T}_{\infty}$ is infinitely divisible; see [35, Prop. 6.2 and 6.3]. Note that a shifted version of $2 \widehat{T}_{\infty}$ has the simple form

$$
\sum_{\ell=1}^{\nu} w_{\ell}\left(E_{\ell}-1\right), \quad w_{\ell}=\frac{1}{\ell-1 / 2}
$$

Similar sums of exponentials appear in works by Simatos, Robert, and Guillemin [33], relative to weights $w_{\ell}=1 / \ell$, as well as by Biane, Pitman, and Yor [1], in the case of quadratic weights, $w_{\ell}=1 / \ell^{2}$.

(iv) A random variable $X$ is self-decomposable if, for each $c \in(0,1)$, there exists a random variable $R_{c}$ such that $X \stackrel{\mathrm{d}}{\equiv} c X^{\prime}+$ $R_{c}$, where $X \stackrel{\mathrm{d}}{=} X^{\prime}$ and $X^{\prime}, R_{c}$ are independent. This property, which is weaker than stability, is known to imply infinite divisibility [35]. 


\section{References}

[1] Biane, P., Pitman, J., AND Yor, M. Probability laws related to the Jacobi theta and Riemann zeta functions, and Brownian excursions. Bulletin of the American Mathematical Society (N.S.) 38, 4 (2001), 435-465.

[2] Billingsley, P. Probability and Measure, 2nd ed. John Wiley \& Sons, 1986.

[3] Cheno, L., Flajolet, P., Françon, J., Puech, C., And Vuillemin, J. Dynamic data structures: Finite files, limiting profiles and variance analysis. In Eighteenth Annual Conference on Communication, Control, and Computing (1980), The University of Illinois at Urbana-Champaign, pp. 223-232.

[4] DE Bruijn, N. G. Asymptotic Methods in Analysis. Dover, 1981. A reprint of the third North Holland edition, 1970 (first edition, 1958).

[5] Diaconis, P. The cutoff phenomenon in finite Markov chains. Proceedings of the National Academy of Sciences of the USA 93 (1996), 1659-1664.

[6] Edelman, A., And Kostlan, E. How many zeros of a random polynomial are real? Bull. Amer. Math. Soc. (N.S.) 32, 1 (1995), 1-37.

[7] Ehrenfest, P., And Ehrenfest, T. Über zwei bekannte Einwände gegen das Boltzmannsche $H$-Theorem. Physikalische Zeitschrift 8, 9 (1907), 311-314.

[8] Euler, L. De fractionibus continuis observationes. Commentarii academiae scientiarum Petropolitanae 11 (1750), 32-81. Reprinted in Opera Omnia: Series 1, Volume XIV, pp. 291-349. Available electronically from the Euler Archive at Dartmouth (E-123).

[9] Feinsilver, P., AND KociK, J. Krawtchouk polynomials and Krawtchouk matrices. In Recent Advances in Applied Probability, R. B.-Y. et al., Ed. Springer Verlag, 2005, pp. 115-141.

[10] FILL, J. A. The passage time distribution for a birth-and-death chain: Strong stationary duality gives a first stochastic proof. arXiv:0707.4042, 2007. Preprint, 14 pages.

[11] Flajolet, P. Combinatorial aspects of continued fractions. Discrete Mathematics 32 (1980), $125-161$. Reprinted in the 35th Special Anniversary Issue of Discrete Mathematics, Volume 306, Issue 10-11, Pages 992-1021 (2006).

[12] Flajolet, P., Dumas, P., and Puyhaubert, V. Some exactly solvable models of urn process theory. Discrete Mathematics \& Theoretical Computer Science (Proceedings) AG (2006), 59-118.

[13] Flajolet, P., And Sedgewick, R. Analytic Combinatorics. Cambridge University Press, 2008. In press; 811 p.+xii; available electronically from the authors' home pages.

[14] Friedman, B. A simple urn model. Communications in Pure and Applied Mathematics 2 (1949), 59-70.

[15] Goulden, I. P., And JaCkson, D. M. Combinatorial Enumeration. John Wiley, New York, 1983.

[16] Goulden, I. P., And Jackson, D. M. Distributions, continued fractions, and the Ehrenfest urn model. Journal of Combinatorial Theory. Series A 41, 1 (1986), 21-31.

[17] Graham, R. L., Knuth, D. E., And Patashnik, O. Concrete Mathematics. Addison Wesley, 1989.

[18] Hwang, H.-K., Kuba, M., And Panholzer, A. Analysis of some exactly solvable diminishing urn models. In Formal Power Series and Algebraic Combinatorics (Tianjin, China, 2007). 12 pages.

[19] JAnson, S. Functional limit theorems for multitype branching processes and generalized Pólya urns. Stochastic Processes and Applications 110, 2 (2004), 177-245.

[20] JAnson, S. Limit theorems for triangular urn schemes. Probability Theory and Related Fields 134 (2006), 417-452. 
[21] Johnson, N. L., And Kotz, S. Urn Models and Their Application. John Wiley, 1977.

[22] KAC, M. Random walk and the theory of Brownian motion. American Mathematical Monthly 54 (1947), 369-391.

[23] KeILson, J. Log-concavity and log-convexity in passage time densities of diffusion and birth-death processes. Journal of Applied Probability 8 (1971), 391-198.

[24] Keilson, J. Markov Chain Models—Rarity and Exponentiality. Springer Verlag, Berlin, 1979.

[25] Kingman, J. F. C. Martingales in the OK Corral. The Bulletin of the London Mathematical Society 31, 5 (1999), 601-606.

[26] Kingman, J. F. C. Stochastic aspects of Lanchester's theory of warfare. Journal of Applied Probability 39, 3 (2002), 455-465.

[27] Kingman, J. F. C., And Volkov, S. E. Solution to the OK Corral model via decoupling of Friedman's urn. Journal of Theoretical Probability 16, 1 (2003), 267-276.

[28] Lukacs, E. Characteristic Functions. Griffin, London, 1970.

[29] Olver, F. W. J. Asymptotics and Special Functions. Academic Press, 1974.

[30] Pemantle, R. A survey of random processes with reinforcement. Probability Surveys 4 (2007), 1-79.

[31] Puyhaubert, V. Modèles d'urnes et phénomènes de seuil en combinatoire analytique. PhD thesis, École Polytechnique, Palaiseau, France, 2005.

[32] Rogers, L. J. On the representation of certain asymptotic series as convergent continued fractions. Proceedings of the London Mathematical Society (Series 2) 4 (1907), 72-89.

[33] Simatos, F., Robert, P., And Guillemin, F. A queueing system for modeling a file sharing principle. arXiv:0803.1610, 2008. 16 pages. To appear in Proceedings of SIGMETRICS'08, June 2-6, 2008, Annapolis, Maryland, USA.

[34] Stanley, R. P. Enumerative Combinatorics, vol. II. Cambridge University Press, 1999.

[35] STEUTEL, F. W., AND VAN HARN, K. Infinite divisibility of probability distributions on the real line. No. 259 in Monographs and Textbooks in Pure and Applied Mathematics. Marcel Dekker, 2004.

[36] Stieltjes, T. Sur la réduction en fraction continue d'une série procédant selon les puissances descendantes d'une variable. Annales de la Faculté des Sciences de Toulouse 4 (1889), 1-17.

[37] Whittaker, E. T., And Watson, G. N. A Course of Modern Analysis, fourth ed. Cambridge University Press, 1927. Reprinted 1973.

[38] Williams, D. Probability with martingales. Cambridge Mathematical Textbooks. Cambridge University Press, Cambridge, 1991.

[39] Williams, D., AND MCIlroy, P. The OK Corral and the power of the law (a curious Poisson-kernel formula for a parabolic equation). The Bulletin of the London Mathematical Society 30, 2 (1998), 166-170. 
